\title{
RACSAM
}

Rev. R. Acad. Cien. Serie A. Mat.

VOL. 103 (1), 2009, pp. 87-90

Análisis Matemático / Mathematical Analysis

\section{About an example of a Banach space not weakly $K$-analytic}

\author{
J. Kąkol and M. López Pellicer
}

\begin{abstract}
Cascales, Kạkol, Saxon proved [3] that in a large class $\mathfrak{G}$ of locally convex spaces (lcs) (containing $(L M)$-spaces and $(D F)$-spaces) for a lcs $E \in \mathfrak{G}$ the weak topology $\sigma\left(E, E^{\prime}\right)$ of $E$ has countable tightness iff its weak dual $\left(E^{\prime}, \sigma\left(E^{\prime}, E\right)\right)$ is $K$-analytic. Applying examples of Pol [9] (and Kunen [12]) one gets that there exist Banach spaces $C(X)$ over a compact scattered space $X$ such that $C(X)$ is not weakly $K$-analytic (even not weakly $K$-countably determined under $(\mathrm{CH})$ ) but the weak dual of $C(X)$ has countable tightness. This provides also an example showing that $(g D F)$-spaces need not be in class $\mathfrak{G}$.
\end{abstract}

\section{Sobre un ejemplo de un espacio de Banach no débilmente $K$-analítico}

Resumen. Cascales, Ka̧kol, Saxon probaron en [3] que en la amplia clase $\mathfrak{G}$ de espacios localmente convexos, que contienen a los espacios $(L M)$ y a los espacios $(D F)$, se tiene que un espacio $E \in \mathfrak{G}$ verifica que su topología débil $\sigma\left(E, E^{\prime}\right)$ tiene tightness numerable si y sólo si su dual débil $\left(E^{\prime}, \sigma\left(E^{\prime}, E\right)\right)$ es $K$-analitico. Aplicando ejemplos de Pol [9] (y de Kunen [12]) se obtiene que existen compactos diseminados (scattered) $X$ tales que el espacio de Banach $C(X)$ no es débilmente $K$-analitico (y ni siquiera es débilmente $K$-numerablemente determinado, si se admite la hipótesis del continuo), pero el dual débil de $C(X)$ tiene tightness numerable, lo que proporciona de exemplo de espacio $(D F)$ generalizado (espacio $(g D F))$ que no pertenece a la clase $\mathfrak{G}$.

\section{Introduction}

Many concrete locally convex spaces (lcs) $E$ are weakly realcompact, i.e. the weak topology $\sigma\left(E, E^{\prime}\right)$ is realcompact. For example any lcs $E$ whose strong dual is metrizable and separable has this property. This follows from (*): If $\left(E, E^{\prime}\right)$ is a dual pair and $\sigma\left(E, E^{\prime}\right)$ has countable tightness (i.e. for every $A \subset E$ and $x \in \bar{A}$ there exists countable $B \subset A$ such that $x \in \bar{B})$, then $\sigma\left(E^{\prime}, E\right)$ is realcompact. Indeed, by Corson [4], see also [17, p.137], it is enough to show that every linear functional $f$ on $E$ which is $\sigma\left(E, E^{\prime}\right)$ continuous on each $\sigma\left(E, E^{\prime}\right)$-closed separable vector subspace is continuous. Note that $K:=f^{-1}(0)$ is closed in $\sigma\left(E, E^{\prime}\right)$ : If $y \in \bar{K}$, then there is countable $D \subset K$ with $y \in \bar{D}$. By assumption $f(\overline{\operatorname{lin}(D)}$ is $\sigma\left(E, E^{\prime}\right)$-continuous, so $f(y) \in \overline{f(\operatorname{lin}(D))} \subset \overline{\operatorname{lin} f(K)}=\{0\}$. Hence $y \in K$, so $f$ is continuous.

The following is much less evident: Every Banach space with Corson property $(C)$ is weakly realcompact, $[4,11]$. It turns out that in a class $\mathfrak{G}$ of lcs (containing all $(L M)$-spaces and $(D F)$-spaces) the converse to $(*)$ holds even in a stronger form [3].

Proposition 1 If $E \in \mathfrak{G}$, then $E_{\sigma}:=\left(E, \sigma\left(E, E^{\prime}\right)\right)$ has countable tightness iff $E_{\sigma}^{\prime}:=\left(E^{\prime}, \sigma\left(E^{\prime}, E\right)\right)$ is K-analytic.

Presentado por / Submitted by Darío Maravall Casesnoves.

Recibido / Received: 2 de marzo de 2009. Aceptado / Accepted: 4 de marzo de 2009.

Palabras clave / Keywords: Banach $C(X)$ space, compact scattered, countable tightness, $K$-analytic, $K$-countable determined, locally convex space, weak topology.

Mathematics Subject Classifications: 46A03, 46E15, 54C30, 54H05.

(C) 2009 Real Academia de Ciencias, España. 
Is it true that a Banach space $E$ is weakly Lindelöf if $E_{\sigma}^{\prime}$ has countable tightness? If $\sigma\left(E, E^{\prime}\right)$ is Lindelöf, is the unit ball in $E^{\prime}$ of countable tightness in $\sigma\left(E^{\prime}, E\right)$ ? This problem is strictly related with Banach spaces satisfying the Corson property $(C)$, i.e. every collection of closed convex subsets of $E$ with empty intersection contains a countable subcollection with empty intersection [4]. Every Banach space $E$ which is $\sigma\left(E, E^{\prime}\right)$-Lindelöf has property $(C)$; the converse fails, see [11] and references.

Pol [11] proved that a Banach space $E$ has property $(C)$ iff $E_{\sigma}^{\prime}$ has property $\left(C^{\prime}\right)$, i.e. for every $A \subset E^{\prime}$ and $f \in \bar{A}$ (the closure in $\sigma\left(E^{\prime}, E\right)$ ) there exists a countable set $B \subset A$ with $f \in \overline{\operatorname{conv} B}$. Still it is unknown if property $\left(C^{\prime}\right)$ can be replaced by countable tightness of the unit ball in $\sigma\left(E^{\prime}, E\right)$, see $[11,5]$. The aim of this short note is to prove Proposition 2 yielding Example 1 and Example 2 stated in Abstract, which answer also a question of Professor P. Domański (personal communication). This applies to show that $(g D F)$-spaces need not to be in class $\mathfrak{G}$.

A lcs $E$ belongs to class $\mathfrak{G}$ if there is a family $\left(A_{\alpha}\right)_{\alpha \in \mathbb{N}^{\mathbb{N}}}$ of subsets in $E^{\prime}$ covering $E^{\prime}$ such that $A_{\alpha} \subset A_{\beta}$ for $\alpha \leqslant \beta$ and in each $A_{\alpha}$ sequences are equicontinuous [2]. All ( $\left.L M\right)$-spaces (hence metrizable lcs) and dual metric spaces (hence $(D F)$-spaces) belong to $\mathfrak{G}$ by Examples from [2].

A topological space $X$ is called Lindelö $\Sigma$ (called also $K$-countable determined) if there is an upper semi-continuous (usco) map from a nonempty subset $\Sigma \subset \mathbb{N}^{\mathbb{N}}$ with compact values in $X$ whose union is $X$, where the set of integers $\mathbb{N}$ is discrete and $\mathbb{N}^{\mathbb{N}}$ is endowed with the product topology $[8,1,6]$. If the same holds for $\Sigma=\mathbb{N}^{\mathbb{N}}$, then $X$ is called $K$-analytic.

\section{One Proposition and Example}

For a Tichonov space $X$ by $C_{p}(X)$ we denote the space of continuous realvalued maps on $X$ endowed with the pointwise topology. In [5, Theorem 3.6] it was proved that if $X$ is a compact zero-dimensional space and $C(X)$ is weakly Lindelöf, then the unit ball in the dual $M(X)$ of $C(X)$ has countable tightness in its weak dual topology, and if $X$ is compact and scattered, then $C(X)$ has property $(C)$ iff $X$ has countable tightness [11, Corollary 1].

For compact scattered $X$ we show also the following simple fact which will be used below.

Proposition 2 Let $X$ be a scattered compact space such that $C(X)$ is weakly Lindelöf. Then the weak dual of $C(X)$ has countable tightness.

Proof. By assumption also $C_{p}(X)$ is Lindelöf. Let $\tau_{p}$ and $\tau_{\sigma}:=\sigma\left(C(X), C(X)^{\prime}\right)$ be the topology of $C_{p}(X)$ and the weak topology of $C(X)$, respectively. Let $B$ be the closed unit ball in $C(X)$. Since $X$ is scattered, then $\tau_{p}\left|B=\tau_{\sigma}\right| B$ by [15, Corollary 19.7.7]. But then $\tau_{p}^{n}\left|B^{n}=\tau_{\sigma}^{n}\right| B^{n}$ for each $n \in \mathbb{N}$, where $B^{n}:=\prod_{1<i<n} B$ and $\tau_{p}^{n}$, $\tau_{\sigma}^{n}$ denote the own product topologies on the product $C(X)^{n}:=\prod_{1 \leq i \leq n} C(X)$. Since $X$ as compact and scattered is zero-dimensional [1, Theorem IV.8.6] applies to deduce that $\left(C(X)^{n}, \tau_{p}^{n}\right)$ is Lindelöf for each $n \in \mathbb{N}$. But then $B^{n}$ (closed in $\tau_{p}^{n}$ ) is also Lindelöf. Hence $B^{n}$ is Lindelöf in $\left(C(X)^{n}, \tau_{\sigma}^{n}\right)$ for each $n \in \mathbb{N}$. Since $C(X)^{n}=\bigcup_{m} m B^{n}$ and each $m B^{n}$ is Lindelöf in $\tau_{\sigma}^{n}$, then $\left(C(X)^{n} \tau_{\sigma}^{n}\right)$ is a Lindelöf space for each $n \in \mathbb{N}$. But [1, Theorem II.1.1] implies that $C_{p}\left(\left(C(X), \tau_{\sigma}\right)\right)$ has countable tightness. Consequently $\left(C(X)^{\prime}, \sigma\left(C(X)^{\prime}, C(X)\right) \subset C_{p}\left(C(X), \tau_{\sigma}\right)\right.$ has countable tightness.

Let $\Gamma \subset \Omega$ be the set of all non-limit ordinals and let $\Lambda=\Omega \backslash \Gamma$. Attach to each $\gamma \in \Lambda$ an increasing sequence $\left(s_{\lambda}(n)\right)_{n}$ in $\Gamma$ such that $\lim _{n \rightarrow \infty} s_{\lambda}(n)=\lambda$. Endow the set $\Omega$ with the following topology: The points from $\Gamma$ are isolated and the basic neighbourhoods of $\gamma \in \Lambda$ are of the form $W_{\gamma}(n)=\{\gamma\} \cup$ $\left\{s_{\gamma}(m): m \geq n\right\}$. Let $X_{0}=\Omega \cup\left\{\omega_{1}\right\}$ be the one-point compactification of the locally compact space $\Omega$. Then $X_{0}$ is scattered. By [9] the Banach space $C\left(X_{0}\right)$ is weakly Lindelöf and by [16] it is not a weakly compactly generated (WCG) Banach space. Since every weakly $K$-analytic Banach space $C(X)$ over compact scattered $X$ is (WCG) by [10], then $C\left(X_{0}\right)$ is not weakly $K$-analytic. Using Proposition 2 one gets an example showing that a variant of Proposition 1 fails in the following sense.

Example 1 The weak dual of $C\left(X_{0}\right)$ has countable tightness but the weak topology of $C\left(X_{0}\right)$ is not K-analytic. 
Last example may suggest the following question: Let $X$ be a compact scattered space such that $C(X)$ is a weakly Lindelöf space. Is then $C(X)$ a weakly Lindelöf $\Sigma$-space? The answer is negative:

Under Continuum Hypothesis $(\mathrm{CH})$ Kunen provided and example, see [12, 9], of a scattered compact separable space $X$ with cardinality $\aleph_{1}$ such that $C(X)$ is weakly Lindelöf. From Proposition 2 the weak dual of $C(X)$ has countable tightness. Note that the weak topology of $C(X)$ is not Lindelöf $\Sigma$. It is enough to show that $C_{p}(X)$ is not Lindelöf $\Sigma$. Since $X$ is separable, it is enough to show that $X$ does not have a countable network (or equivalently $X$ is not cosmic, i.e. $X$ is not a continuous image of a metric separable space) owing to [1, Corollary IV.9.9]. Assume that $X$ is cosmic. Then also $C_{p}(X)$ is cosmic by [7, Corollary 4.1.3]. But then $C_{p}(X)$ would be separable yielding on $X$ a weaker metric topology. It is however well-known that a metric compact scattered space is countable, a contradiction. So we have

Example 2 Under $(\mathrm{CH})$ there exists a compact scattered space $X$ such that $C(X)$ is weakly Lindelöf but not weakly Lindelöf $\Sigma$ and the weak dual of $C(X)$ has countable tightness.

As we have mentioned any $(D F)$-space belongs to class $\mathfrak{G}$. A natural generalization of the concept of $(D F)$-spaces is the class of $(g D F)$-spaces. Following Ruess a lcs $(E, \xi)$ is a $(g D F)$-space if it has a fundamental sequence of bounded sets $\left(S_{n}\right)_{n}$ and $\xi$ is the finest locally convex topology on $E$ of all agreeing with $\xi$ on all sets $S_{n}$. If $E$ is a Fréchet space, then the Mackey dual $\left(E^{\prime}, \mu\left(E^{\prime}, E\right)\right)$ is a $(g D F)$ space, [13, 14]. Set $E:=C\left(X_{0}\right)$, where $C\left(X_{0}\right)$ is as above. Assume that $\left(E^{\prime}, \mu\left(E^{\prime}, E\right)\right) \in \mathfrak{G}$. Since the weak dual of $C\left(X_{0}\right)$ has countable tightness by Proposition 2, then Proposition 1 implies that the weak topology of $C\left(X_{0}\right)$ is $K$-analytic, a contradiction. Therefore, although $(g D F)$-spaces seem to be "close" to $(D F)$-spaces, we have

Corollary 1 There exist $(g D F)$-spaces which do not belong to class $\mathfrak{G}$.

Acknowledgement. This research is supported by the project of Ministry of Science and Higher Education, Poland, grant n. N 201274033 for the first named author and by the project MTM200801502 of the Spanish Ministry of Science and Innovation for the two authors.

\section{References}

[1] Arkhangel's KiI, A. V., (1992). Topological function spaces, Math. and its Applications, 78, Kluwer Academic Publishers, Dordrecht Boston London.

[2] Cascales, B. And Orihuela, J., (1987). On Compactness in Locally Convex Spaces, Math. Z., 195, $365-381$.

[3] Cascales, B., KA̧KOl, J. And SAXon, S. A., (2002). Weight of precompact subsets and tightness, J. Math. Anal. Appl., 269, 500-518.

[4] Corson, H. H., (1961). The weak topology of a Banach space, Trans. Amer. Math. Soc., 101, 1-15.

[5] Frankiewicz, R., Plebanek, G. And Ryll-Nardzewki, C., (2001). Between the Lindelöf property and countable tightness, Proc. Amer. Math. Soc., 129, 97-103.

[6] Kubiś, W., OKunev, O. And Szeptycki, P. J., (2006). On some classes of Lindelöf $\Sigma$-spaces, Topol. Appl., 153, 2574-2590.

[7] McCoy, R. A. And Ntantu, I., (1988). Topological Properties of Spaces of Continuous Functions, Lecture Notes in Math.

[8] Nagami, K., (1969). E-spaces, Fund. Math., 61, 169-192.

[9] PoL, R., (1979). A function space $C(X)$ which is weakly Lindelöf but not weakly compactly generated, Studia Math., 64, 279-285.

[10] PoL, R., (1980). A theorem on the weak topology of $C(X)$ for compact scattered $X$, Fund. Math., 106, $135-140$. 
[11] PoL, R., (1980). On a question of H. H. Corson and some related problems, Fund. Math., 49, 143-154.

[12] Roitman, J., (1984). Basic S and L, in Handbook of Set-theoretical Topology, North Holland , 295-326.

[13] RuESs, W., (1977). On the locally convex structure of strict topologies, Math. Z., 153, 179-192.

[14] Ruess, W., (1982). Weakly compact operators and (DF)-spaces, Pacific J. Math., 98, 419-441.

[15] Semadeni, Z., (1971). Banach spaces of continuous functions, Warszawa.

[16] Wage, M. L., (1976). Applications of set theory to analysis and topology, Thesis, University of WisconsinMadison.

[17] Valdivia, M., (1982). Topics in Locally Convex Spaces, North-Holland, Amsterdam.

\section{J. Kạkol}

Faculty of Mathematics and Informatics,

A. Mickiewicz University

61-614 Poznań,

Poland

kakoleamu.edu.pl

\section{López Pellicer}

Depto. de Matemática Aplicada and IMPA, Universidad Politécnica de Valencia, E-46022 Valencia,

Spain

mlopezpe@mat.upv.es 